\title{
Differences in dietary fibre intake and associated familial factors in a longitudinal study at two time points across adolescence
}

\author{
Olivia G Swann ${ }^{\circledR} \odot$, Monique Breslin ${ }^{1}$, Michelle Kilpatrick', Therese A O'Sullivan² and \\ Wendy H Oddy, * \\ 'Menzies Institute for Medical Research, University of Tasmania, Private Bag 23, Hobart, TAS 7000, Australia: \\ ${ }^{2}$ School of Medical and Health Science, Edith Cowan University, Joondalup, Western Australia, Australia
}

Submitted 13 March 2019: Final revision received 1 August 2019: Accepted 6 August 2019

\begin{abstract}
Objective: Dietary fibre is essential for a healthy diet; however, intake is often inadequate. Understanding of sources of dietary fibre and familial factors associated with intake in adolescents is limited, hampering efforts to increase intake. We aimed to determine adequacy of dietary fibre intake in adolescents, examine how intake changes from mid to late adolescence, identify major food sources and explore associations with familial factors.

Design: Dietary fibre intake measured with semi-quantitative FFQ and sources calculated with the AUSNUT database. Familial factors determined by questionnaire. Setting: Western Australian Pregnancy Cohort (Raine) Study.

Participants: Generation 2 adolescents from the 14- $(n$ 1626) and 17-year $(n 835)$ follow-ups.

Results: Mean intake of dietary fibre did not meet national dietary guidelines other than for females aged 14 years. Mean intake of both sexes was lower at 17 years $(23.0(\mathrm{SD} 10.0) \mathrm{g} / \mathrm{d})$ than at 14 years $(24.3(\mathrm{SD} 9.0) \mathrm{g} / \mathrm{d}, P<0.001)$. The quantity of dietary fibre consumed per megajoule also decreased $(2.6(\mathrm{SD} 0.7) \mathrm{g} / \mathrm{MJ}$ at 14 years, 2.5 (SD 0.9) g/MJ at 17 years, $P=0.007$ ). The greatest source of dietary fibre was cereals and grains, followed by fruits, then vegetables. In multivariable mixed-model analysis, female sex, Caucasian race, age 14 years, good family functioning, high level of parental education and high energy intake were independently associated with higher dietary fibre intake.

Conclusions: Our study highlights an age range and characteristics of adolescents lacking in dietary fibre, thereby identifying target populations for interventions to improve dietary fibre intake across adolescence, which would lead to better health.
\end{abstract}

Keywords
Dietary fibre
Adolescence
Fibre sources
Raine Study

Dietary fibre has beneficial effects on gastrointestinal, cardiovascular and metabolic conditions ${ }^{(1-4)}$ and is considered a key component of a healthy diet. Adolescents' dietary fibre intake is inadequate in most of the Western world, including Australia ${ }^{(5,6)}$. This is despite establishment of minimum intake guidelines in most developed regions and countries, including Canada, the USA, Australia, New Zealand, Europe, Ireland and the $\mathrm{UK}^{(5,7-9)}$. Worldwide dietary consumption trends away from fibre-containing foods towards highly processed 'fast foods' likely contribute to the high prevalence of inadequate intake of dietary fibre ${ }^{(10)}$.

While there are data to show how intake changes between adolescence and adulthood ${ }^{(6)}$, how dietary fibre intake changes from mid to late adolescence within the same group is unknown. The dietary fibre intake of adolescents may have long-term implications, as dietary patterns track from adolescence to adulthood ${ }^{(11-13)}$.

Previous data from Australian surveys suggest that the largest food group contributor to dietary fibre in all age groups is breads and cereals, followed by vegetables ${ }^{(14)}$. However, the current contribution of these food groups to Australian adolescents' dietary fibre intake is unknown.

While adolescence is a time when young people tend to gain independent access to food and greater control of their $\operatorname{diet}^{(15)}$, parenting styles, the strength of family relationships (family functioning) and how strictly parents enforce diet have a considerable impact on food intake in children 
and adolescents ${ }^{(16-19)}$. Additionally, factors such as being in a two-parent family and having more highly educated parents have been associated with a healthy dietary pattern $^{(20)}$. These studies demonstrate the influence of parents over their children's diet and that this influence may persist through to young adulthood, highlighting the potential of familial factors to target interventions where they may be most effective to encourage healthy eating behaviours.

Previous research has focused largely on overall diet or fruit and vegetable intake ${ }^{(18,19)}$, with no known studies examining the associations between familial factors and dietary fibre intake specifically. Additionally, the impact of parental influence has not been studied in relation to the transition of adolescents from a more controlled home diet to increasingly available self-chosen alternatives, although there is evidence that parental control and relationships with food can influence the diet of adolescents after they move out of home ${ }^{(21)}$.

The present study in a large, longitudinal adolescent population provided an opportunity to examine food sources and intake of dietary fibre and associated familial factors. The aims were to:

1. determine the adequacy of dietary fibre intakes in a representative sample of Australian adolescents based on dietary guidelines;

2. compare dietary fibre intakes in mid and late adolescence overall and by sex;
3. identify which food groups provide the major sources of dietary fibre during adolescence and how they differ with age and sex; and

4. examine associations between dietary fibre intake and sex and familial factors, and how these associations differ from mid to late adolescence.

\section{Methods}

\section{Participants}

Participant data for the present study were taken from the Western Australian Pregnancy Cohort (Raine) Study. In the study, 2900 pregnant women were recruited between 1989 and 1991, with 2868 live births. The children (Generation 2) and their families were followed up at multiple time points from birth to young adulthood. Detailed cohort information has been published elsewhere ${ }^{(22,23)}$. Data for the $14-$ and 17-year follow-ups were collected between 2003 and 2009. Initial participant numbers were 1864 at the 14 -year follow-up and 1726 at the 17-year follow-up, with 1632 and 1009 individuals respectively with complete dietary data (Fig. 1). After exclusion for those without complete dietary data and variables of interest, 1626 participants remained from the 14-year follow-up and 836 from the 17-year follow-up. Further exclusion of one participant with missing data on sex left 835 participants with data on all variables at both the 14- and 17-year follow-ups.

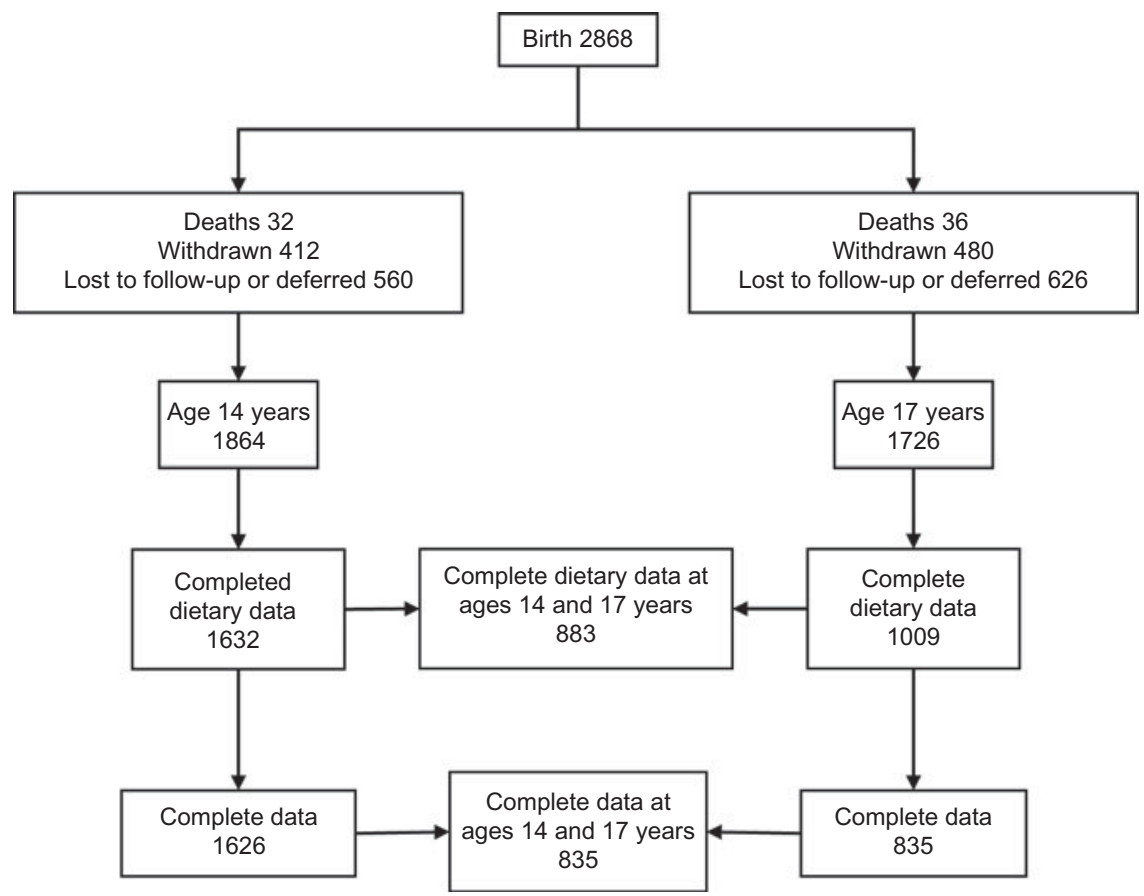

Fig. 1 Number of participants in the Western Australian Pregnancy Cohort (Raine) Study at 14- and 17-year follow-ups and number with complete variables at each age. 'Complete dietary data' includes FFQ items, dietary fibre intake and energy intake. 'Complete data' includes dietary data and all familial factors examined in the present study 


\section{Sociodemographic and familial variables}

Sociodemographic information, including family status, parental employment, financial benefits and household income, was obtained from questionnaires completed by the primary caregiver of the participant at the 14- and 17-year follow-ups. Family status was 'single parent' if only one parent lived at home, or 'two parents' if either both parents or one parent and their partner lived at home. Parental education was surveyed at the 8-year follow-up and the level of educational attainment of the most well-educated parent was selected. Parental race was recorded during pregnancy and the participant's race was categorised as 'Caucasian' if both parents were Caucasian or 'non-Caucasian' if one or both parents were non-Caucasian (Aboriginal, Asian or Polynesian). This is due to interest in cultural differences which may be present even when only one parent is of non-Caucasian race. Family functioning was calculated from the general functioning subscale of the McMaster Family Assessment Device, completed by the primary caregiver at the 14- and 17-year follow-ups ${ }^{(24)}$. The McMaster Family Assessment Device general functioning subscale comprises twelve questions designed to measure family communication and support. Responses are scored between 1 ('strongly agree') and 4 ('strongly disagree'), except for negatively worded questions which are reverse scored. The scores for each of the twelve questions are averaged, with possible total scores ranging between 1 and 4 . Scores of $\geq 2$ are classed as poor family functioning ${ }^{(24)}$.

\section{Dietary variables}

All dietary intake data were obtained from the 212-item Commonwealth Scientific and Industrial Research Organisation (CSIRO) semi-quantitative FFQ. The questionnaire was completed by the primary caregiver together with the adolescent at age 14 years and by the adolescent alone at age 17 years, and measures usual diet over the previous 12 months. Daily intakes of dietary fibre and energy intake per person were provided from the FFQ data, using the Nutrient Tables for use in Australia (NUTTAB) dietary composition database. Dietary fibre intake per megajoule was calculated from those values. The CSIRO FFQ has been shown to be valid and reliable for use in adolescents in the Raine Study and the Australian population ${ }^{(25-27)}$. In the Raine Study population, comparison of the CSIRO FFQ with a $3 \mathrm{~d}$ food record at age 14 years showed a weak to moderate positive correlation for dietary fibre $(r=0.29$ in females and $r=0.43$ in males, $P<0.001$ for both) ${ }^{(25)}$. Similarly, comparison of the CSIRO FFQ with two $4 \mathrm{~d}$ weighed food records in Australian women found no evidence of fixed or proportional bias for dietary fibre with weak to moderate correlation $(r=0.310$, $P<0.05)^{(27)}$

The Australian Food and Nutrient Database (AUSNUT) dietary composition database lists the quantity of dietary fibre in $100 \mathrm{~g}$ of each food item (see online supplementary material, Supplemental Table S1). Items representative of the foods included in the CSIRO FFQ were selected and averages calculated when there was more than one entry for a food item. Dietary fibre concentrations $(\mathrm{g} / 100 \mathrm{~g})$ were combined with the grams of food consumed per day as determined by the FFQ, resulting in an estimated value of dietary fibre obtained per person from each food item. The foods were grouped into cereals and grains, fruits, vegetables, legumes, nuts and discretionary foods. Discretionary foods are prepared foods with added fat or sugar and are unnecessary for a healthy diet. Foods classified as discretionary include biscuits, pizza and cakes. Foods were assigned in the vegetable category based on judgement rather than botanical classification; this allowed the inclusion of fruits such as capsicum, pumpkin and green peas in the vegetable category, as these are widely considered to be vegetables.

The Australian National Health and Medical Research Council has established adequate daily intake levels of dietary fibre for males and females over a range of ages ${ }^{(28)}$. These adequate intake cut-offs are based on the amount of dietary fibre needed for adequate gastrointestinal function and are calculated from median dietary fibre intake in populations where laxation problems are uncommon. Participants in our study were categorised into dietary fibre intake groups based on the Australian guidelines, with an adequate intake of dietary fibre for adolescents at both ages defined as $28 \mathrm{~g} / \mathrm{d}$ for males and $22 \mathrm{~g} / \mathrm{d}$ for females regardless of energy intake ${ }^{(28)}$.

\section{Analytical methods}

Comparison of characteristics of participants in the 14- and 17 -year follow-ups was determined by $\chi^{2}$ tests. Comparison of the Raine Study adolescents' daily dietary fibre intake with the Australian guidelines was achieved with onesample $t$ tests. For the purpose of comparison, dietary fibre intakes were not energy adjusted to maintain consistency with intake guidelines. Daily dietary fibre intake, energy intake and dietary fibre intake per megajoule were compared at the 14- and 17-year follow-ups using paired $t$ tests, both overall and stratified by sex. The proportions of participants in the adequate and inadequate intake categories were examined with $\chi^{2}$ tests.

Linear mixed-effects models were used to determine associations between familial factors and continuous dietary fibre intake at ages 14 and 17 years, including interaction terms to test for differences in associations at the two time points. Familial factors to be included in the model were chosen based on factors identified in the existing literature. The final model was determined with backward stepwise regression and the linear mixed-effects model carried out using random intercepts. Random slopes were tested and not used as they did not significantly add to the model fit. As an interaction between energy intake and 
follow-up age was observed, average marginal effects were calculated using 7 and $11 \mathrm{MJ} / \mathrm{d}$ as examples of low and high energy intakes as they approximately represent the 25th and 75th percentiles. Statistical analysis was performed with $\mathrm{R}$ version $3.5 .0^{(29)}$ and $\mathrm{R}$ studio version $1.1 .453^{(30)}$.

\section{Results}

The majority of the Raine Study participants were Caucasian (88\%). At ages 14 and 17 years, most participants came from a two-parent family that did not receive financial benefits. However, the proportion with parents receiving financial benefits was significantly higher in those attending the 17-year follow-up compared with the 14-year follow-up (31 and 26\%, respectively, $P=0.01$ ). At both follow-ups, their parents were likely to be employed, with at least one parent educated past Year 12 (Table 1). Parental employment and family income were both significantly higher at the 17-year follow-up ( $P<0.001$ for both).

Table 1 Characteristics of adolescents and their families who participated in the 14- and 17-year follow-ups of Western Australian Pregnancy Cohort (Raine) Study

\begin{tabular}{|c|c|c|c|c|c|}
\hline & \multicolumn{2}{|c|}{$\begin{array}{c}\text { Age } 14 \text { years } \\
(n 1626)\end{array}$} & \multicolumn{2}{|c|}{$\begin{array}{c}\text { Age } 17 \text { years } \\
(n 835)\end{array}$} & \multirow[b]{2}{*}{$P$ value } \\
\hline & $n$ & Valid \% & $n$ & Valid \% & \\
\hline \multicolumn{6}{|l|}{ Sex } \\
\hline Female & 791 & $48 \cdot 7$ & 430 & 51.5 & 0.19 \\
\hline \multicolumn{6}{|l|}{ Family status } \\
\hline Two-parent & 1268 & $78 \cdot 1$ & 662 & 80.5 & 0.18 \\
\hline Single-parent & 356 & 21.9 & 160 & $19 \cdot 5$ & \\
\hline \multicolumn{6}{|l|}{ Receive benefits } \\
\hline Yes & 419 & $25 \cdot 9$ & 258 & 30.9 & 0.01 \\
\hline \multicolumn{6}{|c|}{ Highest level of parental education* } \\
\hline No formal qualification & 191 & $12 \cdot 7$ & 80 & $10 \cdot 0$ & 0.06 \\
\hline Year 12 & 150 & $10 \cdot 0$ & 74 & $9 \cdot 3$ & \\
\hline TAFE/trade $\dagger$ & 505 & 33.6 & 253 & 31.7 & \\
\hline University & 658 & $43 \cdot 8$ & 392 & $49 \cdot 1$ & \\
\hline \multicolumn{6}{|c|}{ Primary caregiver employment status } \\
\hline Unemployed & 388 & 23.9 & 130 & $15 \cdot 6$ & $<0.001$ \\
\hline Employed & 1233 & $76 \cdot 1$ & 705 & 84.4 & \\
\hline \multicolumn{6}{|c|}{ Annual family income (\$AU) } \\
\hline$\leq 35000$ & 380 & $23 \cdot 8$ & 107 & $13 \cdot 0$ & $<0.001$ \\
\hline $35001-70000$ & 566 & 35.4 & 201 & 24.5 & \\
\hline $70001-104000$ & 358 & $22 \cdot 4$ & 235 & $28 \cdot 6$ & \\
\hline$>104000$ & 294 & $18 \cdot 4$ & 278 & 33.9 & \\
\hline \multicolumn{6}{|l|}{ Family functioning $\ddagger$} \\
\hline$<2$ & 1048 & $64 \cdot 7$ & 512 & $61 \cdot 7$ & 0.15 \\
\hline$\geq 2$ & 571 & $35 \cdot 3$ & 318 & $38 \cdot 3$ & \\
\hline \multicolumn{6}{|l|}{ Race } \\
\hline Caucasian & 1406 & 88.3 & 723 & 88.2 & 1.00 \\
\hline Non-Caucasian§ & 187 & $11 \cdot 7$ & 97 & $11 \cdot 8$ & \\
\hline \multicolumn{6}{|l|}{ Dietary fibre intake $\|$} \\
\hline Adequate & 678 & $41 \cdot 7$ & 300 & 35.9 & 0.01 \\
\hline
\end{tabular}

Valid \% excludes those with not stated responses.

*Highest level of education of either parent.

†TAFE, Technical and Further Education.

¥Family functioning $<2$ indicates good family relationships while $\geq 2$ indicates potential problematic relationships.

$\S$ Race category 'non-Caucasian' includes Aboriginal, Asian and Polynesian.

|| Adequate intake based on recommended adequate intake of $22 \mathrm{~g} / \mathrm{d}$ for adolescent females and $28 \mathrm{~g} / \mathrm{d}$ for adolescent males ${ }^{(28)}$.
Most participants had a low family functioning score, indicating good family relationships. The proportion of all participants with adequate dietary fibre intake was significantly lower at the 17-year follow-up than at the 14-year follow-up (36 and $42 \%$, respectively, $P=0 \cdot 01$; Table 1).

Mean dietary fibre intake in males at ages 14 and 17 years $(P<0.001$ for both) and females at age 17 years $(P=0.006)$ was significantly lower than the Australian adequate intake guidelines, while mean intake in females at age 14 years was greater than adequate $(P<0 \cdot 001)$. The proportion of females with adequate dietary fibre intake $(>22 \mathrm{~g} / \mathrm{d})$ was significantly lower at age 17 years ( $40 \%)$ than at age 14 years (49\%) in paired analysis $(P=0 \cdot 02)$. The same was seen in males, with the proportion with adequate intake $(>28 \mathrm{~g} / \mathrm{d})$ higher at age 14 years $(35 \%)$ than at 17 years $(32 \%, P<0.001$; Table 2$)$.

In paired analysis (Table 2), overall mean intake of dietary fibre was significantly lower at age 17 years compared with age 14 years $(P<0 \cdot 001)$, as was the quantity of dietary fibre consumed per megajoule $(P=0 \cdot 007)$. However, energy intake did not differ significantly between ages 14 and 17 years $(P=0 \cdot 87)$. In females, there was a significant reduction in mean daily intake of dietary fibre $(P<0.001)$ and energy intake $(P<0.001)$ at age 17 years compared with age 14 years. As a result, the intake per megajoule in females from age 14 to age 17 years was relatively unchanged $(P=0 \cdot 37)$. In males the mean daily dietary fibre intake remained steady between ages 14 and 17 years $(P=0.63)$, while energy intake was significantly higher at age 17 years $(P<0 \cdot 001)$. Consequentially, intake of dietary fibre per megajoule was significantly lower at age 17 years than at age 14 years in males $(P<0.001)$.

Sources of dietary fibre differed from age 14 to 17 years (see online supplementary material, Supplemental Table S1 for detailed information regarding sources of dietary fibre). Overall, adolescents aged 17 years gained more dietary fibre from cereals and grains, less from fruits, and more from vegetables than at age 14 years (Fig. 2(a)). Females obtained less dietary fibre from fruits and discretionary foods and more from vegetables at age 17 years than at age 14 years (Fig. 2(b)), and males consumed more from cereals and grains and less from fruits (Fig. 2(c)). Males consumed more dietary fibre from cereals and grains and discretionary foods than females at ages 14 and 17 years, along with lower intakes from fruits and vegetables (see Supplemental Table S2 for exact proportions and quantities). Sources of dietary fibre also differed between those with adequate and inadequate intakes. Those with adequate intake obtained more dietary fibre from fruits, while those with inadequate intake had a higher intake from cereals and grains, vegetables and discretionary foods (Supplemental Table S3).

In general, the greatest sources of dietary fibre were not foods rich in fibre, but rather foods eaten in the largest quantities. Bran and wheat germ were by far the most 
Dietary fibre intake in adolescents

Table 2 Mean dietary fibre intake at age 14 years and 17 years, overall and by sex (paired), in adolescents participating in the 14- and 17-year follow-ups of the Western Australian Pregnancy Cohort (Raine) Study

\begin{tabular}{|c|c|c|c|c|c|c|c|c|c|c|c|c|c|c|c|}
\hline & \multicolumn{5}{|c|}{ Overall } & \multicolumn{5}{|c|}{ Female } & \multicolumn{5}{|c|}{ Male } \\
\hline & \multicolumn{2}{|c|}{ Age 14 years } & \multicolumn{2}{|c|}{ Age 17 years } & \multirow{2}{*}{$\begin{array}{c}P \\
\text { value }\end{array}$} & \multicolumn{2}{|c|}{ Age 14 years } & \multicolumn{2}{|c|}{ Age 17 years } & \multirow{2}{*}{$\begin{array}{c}P \\
\text { value }\end{array}$} & \multicolumn{2}{|c|}{ Age 14 years } & \multicolumn{2}{|c|}{ Age 17 years } & \multirow{2}{*}{$\begin{array}{c}P \\
\text { value }\end{array}$} \\
\hline & Mean & SD & Mean & SD & & Mean & SD & Mean & SD & & Mean & SD & Mean & SD & \\
\hline$n$ & 880 & & 880 & & & 466 & & 466 & & & 414 & & 414 & & \\
\hline $\begin{array}{l}\text { Total dietary } \\
\text { fibre }(\mathrm{g} / \mathrm{d})\end{array}$ & $24 \cdot 3$ & $9 \cdot 0$ & 23.0 & $10 \cdot 0$ & $<0.001$ & 23.4 & $9 \cdot 1$ & $21 \cdot 1$ & $8 \cdot 6$ & $<0.001$ & $25 \cdot 4$ & $8 \cdot 8$ & $25 \cdot 1$ & $11 \cdot 0$ & 0.63 \\
\hline $\begin{array}{l}\text { Total energy } \\
(\mathrm{MJ} / \mathrm{d})\end{array}$ & 9.56 & 3.15 & 9.58 & 4.07 & 0.87 & $8 \cdot 80$ & 2.96 & 8.00 & $2 \cdot 75$ & $<0.001$ & $10 \cdot 41$ & $3 \cdot 14$ & $11 \cdot 35$ & 4.57 & $<0.001$ \\
\hline \multirow{2}{*}{$\begin{array}{l}\text { Total dietary } \\
\text { fibre (g/MJ) }\end{array}$} & $2 \cdot 6$ & 0.7 & $2 \cdot 5$ & 0.9 & 0.007 & $2 \cdot 7$ & 0.7 & $2 \cdot 7$ & $1 \cdot 0$ & 0.37 & 2.5 & 0.7 & $2 \cdot 3$ & 0.8 & $<0.001$ \\
\hline & $n$ & $\%$ & $n$ & $\%$ & $\begin{array}{c}P \\
\text { value }\end{array}$ & $n$ & $\%$ & $n$ & $\%$ & $\begin{array}{c}P \\
\text { value }\end{array}$ & $n$ & $\%$ & $n$ & $\%$ & $\begin{array}{c}P \\
\text { value } \\
\end{array}$ \\
\hline \multicolumn{16}{|l|}{ Intake level* } \\
\hline Adequate & 372 & 42 & 318 & 36 & $<0.001$ & 229 & 49 & 185 & 40 & 0.02 & 143 & 35 & 133 & 32 & $<0.001$ \\
\hline
\end{tabular}

Three participants with inconsistent sex categorisation were excluded from the analysis.

${ }^{*}$ Adequate intake based on recommended adequate intake of $22 \mathrm{~g} / \mathrm{d}$ for adolescent females and $28 \mathrm{~g} / \mathrm{d}$ for adolescent males ${ }^{(28)}$.

(a)

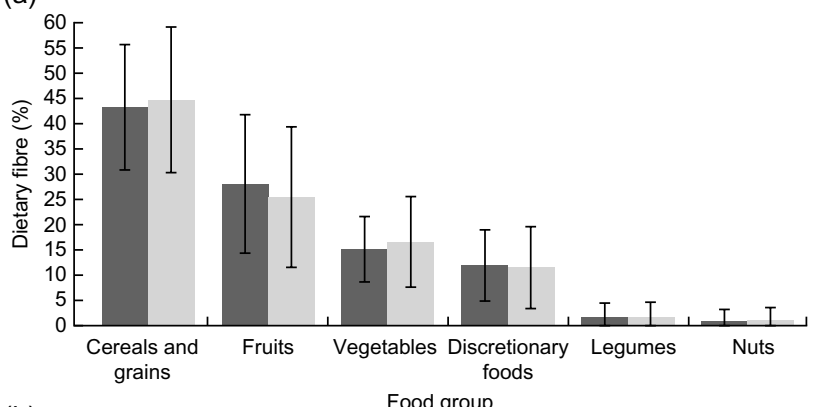

(b)

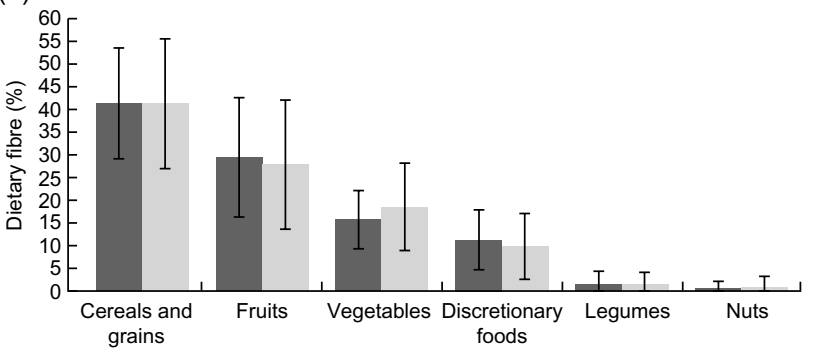

(c)

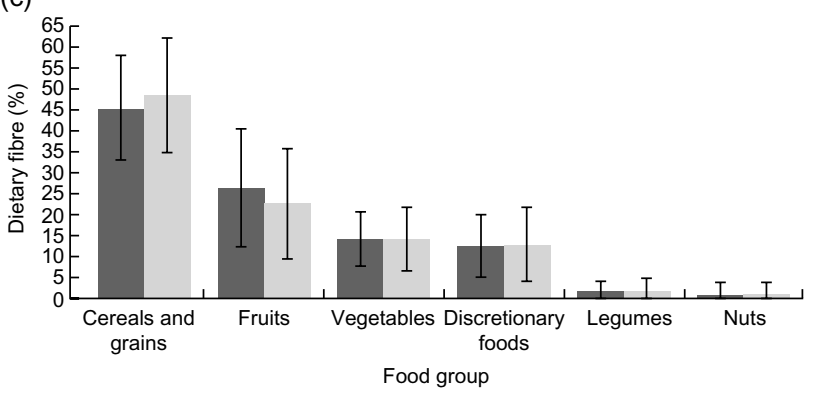

Fig. 2 Percentage of dietary fibre intake obtained from each group of foods at age 14 years $(n 1626 ; \square)$ and age 17 years ( $n 835 ; \quad$ ), overall (a) and in females (b) and males (c) separately, in adolescents participating in the 14- and 17-year follow-ups of the Western Australian Pregnancy Cohort (Raine) Study. Values are means with their standard deviations represented by vertical bars. Discretionary foods include biscuits, pizza and cakes (full food group contents are listed in the online supplementary material, Supplemental Table S1) 
fibre-rich foods at 28 and $19 \%$ fibre content, respectively; however, they were consumed in such small amounts that they contributed very little to dietary fibre intake at ages 14 and 17 years ( $<1 \%$ of daily dietary fibre intake). Conversely, the highest contributor at age 14 years was apples/pears ( $9.3 \%$ of daily dietary fibre intake), not because they are particularly high in dietary fibre (2.6\%), but because they were eaten in the largest quantities. The highest contributor at age 17 years was wholemeal bread ( $12 \cdot 1 \%$ of daily dietary fibre intake), with a comparatively higher fibre content of $7 \cdot 1 \%$ (see online supplementary material, Supplemental Table S1 for full list of food contributions to daily intake).

In mixed-model analysis in which models of fibre intake and familial factors were adjusted only for energy intake (Table 3), low dietary fibre intake was associated with male sex, a high (poor) family functioning score, being aged 17 years and non-Caucasian race. Compared with those adolescents whose parents had no formal qualifications, having at least one parent with a Year 12 education or a university education was associated with higher dietary

Table 3 Mixed-model analysis of associations of familial factors with dietary fibre intake, adjusted only for energy intake, in adolescents participating in the 14- and 17-year follow-ups of the Western Australian Pregnancy Cohort (Raine) Study

\begin{tabular}{|c|c|c|c|}
\hline & $\beta$ & $95 \% \mathrm{Cl}$ & $P$ value \\
\hline$n^{*}$ & 2461 & & \\
\hline \multicolumn{4}{|l|}{ Sex } \\
\hline Female & Reference & & \\
\hline Male & -1.64 & $-2.30,-0.99$ & $<0.001$ \\
\hline \multicolumn{4}{|l|}{ Family status } \\
\hline Two-parent & Reference & & \\
\hline Single-parent & -0.64 & $-1.37,0.09$ & 0.088 \\
\hline \multicolumn{4}{|l|}{ Receive benefits } \\
\hline No & Reference & & \\
\hline Yes & -0.62 & $-1.25,0.00$ & 0.052 \\
\hline \multicolumn{4}{|l|}{ Parental education } \\
\hline No formal qualification & Reference & & \\
\hline Year 12 & $2 \cdot 22$ & $0.86,3.58$ & 0.001 \\
\hline TAFE/trade & 1.05 & $-0.01,2 \cdot 11$ & 0.054 \\
\hline University education & 3.57 & $2 \cdot 54,4.60$ & $<0.00$ \\
\hline \multicolumn{4}{|c|}{ Primary caregiver employment status } \\
\hline Employed & Reference & & \\
\hline Unemployed & 0.31 & $-0.39,1.02$ & 0.382 \\
\hline \multicolumn{4}{|l|}{ Annual family income (\$AU) } \\
\hline$\leq 35000$ & Reference & & \\
\hline $35001-70000$ & 0.11 & $-0.70,0.92$ & 0.787 \\
\hline $70001-104000$ & 0.37 & $-0.50,1.23$ & 0.408 \\
\hline$>104000$ & 1.02 & $0.11,1.92$ & 0.027 \\
\hline Family functioning $\ddagger$ & -1.74 & $-2 \cdot 39,-1.09$ & $<0.001$ \\
\hline \multicolumn{4}{|l|}{ Age of assessment } \\
\hline 14-year follow-up & Reference & & \\
\hline 17-year follow-up & $-1 \cdot 17$ & $-1.65,-0.68$ & $<0.00$ \\
\hline \multicolumn{4}{|l|}{ Race } \\
\hline Caucasian & Reference & & \\
\hline Non-Caucasian & -1.01 & $-2.00,-0.03$ & 0.04 \\
\hline Energy intake (MJ) & 1.89 & $1.81,1.97$ & $<0.00$ \\
\hline
\end{tabular}

*1626 observations from the 14-year follow-up and 835 from the 17-year follow-up contributed to the model.

†TAFE, Technical and Further Education.

$\ddagger$ A measure of family relationships, with higher scores indicating poorer family functioning.
Table 4 Multivariable mixed-model analysis of associations of familial factors with dietary fibre intake in adolescents participating in the 14- and 17-year follow-ups of the Western Australian Pregnancy Cohort (Raine) Study

\begin{tabular}{|c|c|c|c|}
\hline & $\beta$ & $95 \% \mathrm{Cl}$ & $P$ value \\
\hline$n^{*}$ & 2461 & & \\
\hline \multicolumn{4}{|l|}{ Sex } \\
\hline Female & Reference & & \\
\hline Male & -1.54 & $-2 \cdot 20,-0.88$ & $<0.001$ \\
\hline \multicolumn{4}{|l|}{ Parental education } \\
\hline No formal qualification & Reference & & \\
\hline Year 12 & 2.45 & $1 \cdot 10,3 \cdot 79$ & $<0.001$ \\
\hline TAFE/tradet & $1 \cdot 13$ & $0.08,2.18$ & 0.035 \\
\hline University education & 3.66 & $2 \cdot 65,4.68$ & $<0.001$ \\
\hline Family functioning $\ddagger$ & $-1 \cdot 75$ & $-2 \cdot 40,-1.09$ & $<0.001$ \\
\hline \multicolumn{4}{|c|}{ Age of assessment, 17-year v. 14-year } \\
\hline $7 \mathrm{MJ} / \mathrm{d}$ & -0.36 & $-0.97,0.24$ & 0.240 \\
\hline $11 \mathrm{MJ} / \mathrm{d}$ & -1.59 & $-2 \cdot 11,-1 \cdot 07$ & $<0.001$ \\
\hline \multicolumn{4}{|l|}{ Race } \\
\hline Caucasian & Reference & & \\
\hline Non-Caucasian & $-1 \cdot 17$ & $-2 \cdot 15,-0 \cdot 19$ & 0.020 \\
\hline \multicolumn{4}{|l|}{ Energy intake } \\
\hline $1 \mathrm{MJ}$ increase (14-year) & 2.02 & $1 \cdot 92,2 \cdot 12$ & $<0.001$ \\
\hline $1 \mathrm{MJ}$ increase (17-year) & 1.71 & $1.60,1.83$ & $<0.001$ \\
\hline
\end{tabular}

*1626 observations from the 14-year follow-up and 835 from the 17-year follow-up contributed to the model.

†TAFE, Technical and Further Education.

$\ddagger A$ measure of family relationships, with higher scores indicating poorer family functioning.

fibre intake, as was having an annual family income of >\$AU 104000 and a higher energy intake.

In adjusted analysis (Table 4), associations of male sex, a high (poor) family functioning score and non-Caucasian race with a low dietary fibre intake remained significant. Having at least one parent with a Technical and Further Education/trade education, a Year 12 education or a university education, and having a higher energy intake, were associated with greater dietary fibre intake. A significant negative interaction between energy intake and followup age was observed. Average marginal effects for age for 7 and $11 \mathrm{MJ} / \mathrm{d}$ are reported in Table 4. This interaction has two consequences for interpretation. One is that being aged 17 years was associated with a lower dietary fibre intake, but the effect was only large enough to be significant in those with higher energy intake. The other is that diets were significantly less fibre-dense at age 17 years compared with age 14 years.

\section{Discussion}

In the present study of Australian adolescents, we aimed to examine dietary fibre intake including sources and associated familial factors. We found that average daily dietary fibre intake was inadequate for males at ages 14 and 17 years, and for females at age 17 years. Mean intakes were lower at age 17 years than at age 14 years in all adolescents combined, and in females specifically. However, dietary fibre intake remained unchanged in males. The 
greatest contributor to fibre consumption in the Raine Study adolescents at all ages and genders was cereals and grains, followed by fruits, vegetables, discretionary foods (e.g. biscuits, pizza, cakes), and finally legumes and nuts as the lowest contributors. Poor family functioning, non-Caucasian race, being aged 17 years and male sex were associated with lower dietary fibre intake, while a high intake was associated with increasing levels of parental education and high energy consumption.

The inadequate dietary fibre intake seen in the majority of 14- and 17-year-old adolescents in the present study is consistent with what is observed in the Australian population and much of the Western world ${ }^{(5,6)}$. Other research analysing 2011-2012 national survey data of the Australian population showed that less than $30 \%$ of adolescents aged 14-18 years met the dietary fibre intake guidelines ${ }^{(6)}$. While the proportion of Raine Study adolescents meeting the guidelines (between 33 and 50\% depending on age and sex) was higher than was seen in the national survey data, this may be due to potential overestimation of dietary fibre intake by the FFQ as seen in validation studies ${ }^{(25,27)}$. It is worth noting that these Australian adequate intake quantities are based solely on gastrointestinal function. Thus, adequacy as determined by these guidelines may not be sufficient to provide the other benefits associated with dietary fibre such as reduced blood cholesterol. Additionally, these dietary fibre intake recommendations were not published until 2006. Although 2006 was the start of the 17-year follow-up, the FFQ is retrospective and therefore the impact of recommendations on dietary fibre intake was not measured in our study.

In the present study, diets became less fibre-dense from mid to late adolescence, as both dietary fibre intake and dietary fibre intake per megajoule were lower at age 17 years than at age 14 years. In females the lower dietary fibre intake at age 17 years can be explained by a concurrent reduction in energy intake. Conversely, due to an increase in energy intake between the ages of 14 and 17 years, the daily dietary fibre intake for males did not change. These results suggest a need for different strategies to address dietary fibre intake in males and females, as the underlying reason for the inadequate intake may differ.

Australian data from 1995 show that in adolescents aged 12-18 years, approximately $45 \%$ of dietary fibre intake came from breads and cereals, $10 \%$ from fruit, $25 \%$ from vegetables and $2 \%$ from legumes ${ }^{(14)}$. In our study, the contribution of cereals and grains to fibre intake was relatively unchanged (41-45\%); however, the contribution from vegetables was lower (14-18\%) than in the data from 1995 and intake from fruits more than doubled (22-29\%). On the whole, adolescents in our study gained more dietary fibre from low-fibre foods than from high-fibre foods, mostly due to the higher quantities of low-fibre foods consumed.

The finding that poor family functioning, representing a lack of family communication and support, was associated with lower dietary fibre intake in this sample of adolescents is supported by research in children showing that poor family functioning was associated with lower consumption of fruits and vegetables ${ }^{(19)}$. As we have shown, fruits and vegetables are important sources of dietary fibre for our participants, contributing between 37 and $47 \%$ of daily dietary fibre intake dependent on age and sex. Poor family functioning has been associated with a deficiency or overabundance of independence, and strict or unstructured discipline, among other factors ${ }^{(31)}$. The association between poor family functioning and a lower dietary fibre intake found in our study could be due to a lack of supervision, as it has been shown that the presence of parents at meals is associated with higher fibre intake and lower risk of reduced fruit and vegetable intakes ${ }^{(32,33)}$. Additionally, overly strict parenting could play a role, as a restrictive diet can result in overeating and poor self-regulation of intake in children $^{(16,17)}$.

Other factors associated with fibre intake were race, sex and parental education. Non-Caucasian race was associated with lower intake of dietary fibre, potentially because of differences in diet related to cultural heritage ${ }^{(32)}$. The association between male sex and lower intake of dietary fibre was in contrast to the observation that dietary fibre intake was higher in males than in females at both ages. However, this difference can be explained by the adjustment for energy intake, consistent with our results and the literature showing that females ate more dietary fibre per megajoule than males ${ }^{(34,35)}$. The finding that high dietary fibre intake was associated with increasing levels of parental education is consistent with previous research showing that adolescents with more highly educated parents were more likely to have a nutritious $\operatorname{diet}^{(32)}$.

There are a number of strengths and limitations to our study. A major strength is the use of a large populationbased study with comprehensive participant data. The 14- and 17-year follow-ups allowed us to track dietary fibre intakes within matched samples to analyse the changes to intake over time. The use of the FFQ is advantageous as it is not affected by transient changes in diet and reflects normal intake over the past 12 months. However, there remains potential for errors in the calculation of nutrient quantity due to incorrect estimation of portion size and the questionnaire may be subject to recall bias. The FFQ was completed in conjunction with the primary caregiver at age 14 years, to improve accuracy of reporting, and by the participant only at age 17 years. This may have led to differences in reporting, as it is possible that the jointly filled-out 14-year follow-up questionnaire was subject to social desirability bias from the adolescent or parent ${ }^{(36)}$. As such, the 17-year FFQ may be a more accurate representation of diet. Additionally, the dietary fibre quantification method used in the FFQ (AOAC enzymatic-gravimetric method 985.29)(37) may underestimate dietary fibre intake compared with the methods used in the development of the adequate intake recommendations. Conversely, 
validation studies against other methods of diet measurement suggest that the CSIRO FFQ may overestimate dietary fibre intake $\mathrm{e}^{(25,27)}$.

There were limitations in the determination of dietary fibre sources in the present study. The food composition database used to determine the dietary fibre content of foods (AUSNUT) was not the same as that used by the CSIRO (NUTTAB), and therefore estimations of dietary fibre content may vary slightly from those used by the CSIRO when calculating daily dietary fibre intakes. However, both the AUSNUT and NUTTAB used the same analytical techniques and contain composition data from a wide range of food items, allowing means to be obtained from multiple entries to accurately estimate dietary fibre content. There also may have been slight differences in the classification of foods. Fruits and vegetables can be somewhat fluid in categorisation, with some fruits such as capsicum, pumpkin and green peas commonly considered vegetables. Additionally, the type of breakfast cereal consumed was not considered when calculating a mean dietary fibre content of breakfast cereal, which may have led to inaccuracies in the contribution of breakfast cereal to dietary fibre intake. However, estimation of dietary fibre content based on the most frequently eaten cereals suggests that any inaccuracies would be minor.

The data from the 14- and 17-year follow-ups of Raine Study adolescents were collected between 2003 and 2009, and therefore may not represent diets of adolescents today. However, research in America has shown that while childhood and adolescent dietary fibre intakes have been slowly increasing over the last 50 years, they have remained consistently inadequate ${ }^{(38,39)}$. Furthermore, data from the most recent Australian national nutrition survey (2011-2012) show inadequate adolescent dietary fibre intakes, with mean intakes lower than those observed in the present study ${ }^{(6)}$.

By identifying an age range and characteristics of adolescents who are lacking in dietary fibre, our study provides information to help guide interventions to improve dietary fibre intake. Understanding of current intakes can be used to build strategies to increase them, leading to improved health. For example, our findings suggest that additional exposure to the benefits of dietary fibre may be advantageous for older male adolescents, as their diets are the least fibre-dense. Furthermore, encouraging older adolescents to maintain a higher intake of fruits may be an effective method of increasing fibre intake, as it was the food group which had the largest reduction in intake at age 17 years compared with age 14 years. Additionally, the finding that most dietary fibre comes from low-fibre foods, and that high-fibre foods are not eaten in great quantities in this age group, suggests a general preference for low-fibre foods. Therefore, research into methods to increase the fibre content of already commonly eaten foods without reducing their palatability may be an alternative method to increase dietary fibre intake.

\section{Acknowledgements}

Acknowledgements: The authors are grateful to the Western Australian Pregnancy Cohort (Raine) Study participants and their families, and thank the Raine Study research staff for cohort coordination and data collection. The core management of the Raine Study is funded by the University of Western Australia, Curtin University, Telethon Kids Institute, Women and Infants Research Foundation, Edith Cowan University, Murdoch University, the University of Notre Dame Australia and the Raine Medical Research Foundation. The authors thank the National Health and Medical Research Council of Australia (NHMRC) for its long-term contribution to funding the study over the last 29 years and the Telethon Kids Institute for long-term support of the Raine Study. Financial support: This work was supported by the Heart Foundation Beyond Blue Strategic Research Program (W.H.O. et al., ID G08P4036 2009-2012). The Raine Study received funding from the Raine Medical Research Foundation at the University of Western Australia, the NHMRC, the Telstra Research Foundation, the Western Australian Health Promotion Foundation and the Australian Rotary Health Research Fund. The authors acknowledge the Telethon Kids Institute, the Commonwealth Scientific and Industrial Research Organization, the NHMRC Program Grant ID \#003209 and Project Grant \#211912 for supporting the 14-year follow up. Data collection and biological specimens at the 17-year follow-up were funded by the NHMRC Program Grant ID 353514 and Project Grant \#403981. The funders had no role in the design, analysis or writing of this article. Conflict of interest: None. Authorship: O.G.S. contributed in conception, design, analysis, interpretation, drafting and preparation of the final manuscript. M.B. contributed in conception, design, analysis, interpretation, drafting and review of the final manuscript. M.K. contributed in conception, design, drafting and review of the final manuscript. T.A.O. contributed in drafting, interpretation and review of the final manuscript. W.H.O. contributed in conception, design, analysis, interpretation, drafting and review of the final manuscript. Ethics of buman subject participation: The Raine Study was conducted according to the guidelines laid down in the Declaration of Helsinki and was approved by the ethics committees of King Edward Memorial Hospital for Women and Princess Margaret Hospital for Children, Perth, Western Australia. Informed consent was obtained from the adolescent and their primary caregiver at each follow-up.

\section{Supplementary material}

To view supplementary material for this article, please visit https://doi.org/10.1017/S1368980019003446 


\section{References}

1. Streppel MT, Ocke MC, Boshuizen HC et al. (2008) Dietary fiber intake in relation to coronary heart disease and all-cause mortality over 40 y: the Zutphen Study. Am J Clin Nutr $\mathbf{8 8}$, 1119-1125.

2. Meyer KA, Kushi LH, Jacobs DR Jr et al. (2000) Carbohydrates, dietary fiber, and incident type 2 diabetes in older women. Am J Clin Nutr 71, 921-930.

3. Anderson JW, Baird P, Davis RH Jr et al. (2009) Health benefits of dietary fiber. Nutr Rev 67, 188-205.

4. Gianfredi V, Salvatori T, Villarini M et al. (2018) Is dietary fibre truly protective against colon cancer? A systematic review and meta-analysis. Int J Food Sci Nutr 69, 904-915.

5. Edwards CA, Xie C \& Garcia AL (2015) Dietary fibre and health in children and adolescents. Proc Nutr Soc $\mathbf{7 4}$ 292-302.

6. Fayet-Moore F, Cassettari T, Tuck K et al. (2018) Dietary fibre intake in Australia. Paper I: associations with demographic, socio-economic, and anthropometric factors. Nutrients 10, 599 .

7. Australian Bureau of Statistics (2015) Australian Health Survey: Usual Nutrient Intakes, 2011-12. http://www.abs.gov.au/ AUSSTATS/abs@.nsf/DetailsPage/4364.0.55.0082011-12? OpenDocument (accessed March 2019).

8. EFSA Panel on Dietetic Products, Nutrition and Allergies (2010) Scientific opinion on dietary reference values for carbohydrates and dietary fibre. EFSA J 8, 1462.

9. US Department of Agriculture \& US Department of Health and Human Services (2009) What We Eat in America. National Health and Nutrition Examination Survey (NHANES) 2003-2006. Dietary fiber: usual intakes from food and water, 2003-2006, compared to adequate intakes. https:// www.ars.usda.gov/ARSUserFiles/80400530/pdf/0506/usual_ nutrient_intake_dietary_fiber_2003-06.pdf (accessed March 2019).

10. Popkin BM (2006) Global nutrition dynamics: the world is shifting rapidly toward a diet linked with noncommunicable diseases. Am J Clin Nutr 84, 289-298.

11. Branen L \& Fletcher J (1999) Comparison of college students' current eating habits and recollections of their childhood food practices. J Nutr Educ 31, 304-310.

12. Lien N, Lytle LA \& Klepp K-I (2001) Stability in consumption of fruit, vegetables, and sugary foods in a cohort from age 14 to age 21. Prev Med 33, 217-226.

13. Movassagh EZ, Baxter-Jones ADG, Kontulainen S et al. (2017) Tracking dietary patterns over 20 years from childhood through adolescence into young adulthood: the Saskatchewan pediatric bone mineral accrual study. Nutrients $\mathbf{9}, 990$.

14. McLennan W \& Podger A (1998) National Nutrition Survey: Nutrient Intakes and Physical Measurements, Australia, 1995. http://www.ausstats.abs.gov.au/ausstats/subscriber. nsf/0/CA25687100069892CA25688900268A6D/\$File/48050_ 1995.pdf (accessed March 2019).

15. Story M, Neumark-Sztainer D \& French S (2002) Individual and environmental influences on adolescent eating behaviors. J Am Diet Assoc 102, 3 Suppl., S40-S51.

16. Faith MS, Scanlon KS, Birch LL et al. (2004) Parent-child feeding strategies and their relationships to child eating and weight status. Obes Res 12, 1711-1722.

17. Fisher JO \& Birch LL (1999) Restricting access to palatable foods affects children's behavioral response, food selection, and intake. Am J Clin Nutr 69, 1264-1272.

18. Kremers SPJ, Brug J, de Vries H et al. (2003) Parenting style and adolescent fruit consumption. Appetite 41, 43-50.

19. Renzaho AM, Kumanyika S \& Tucker KL (2011) Family functioning, parental psychological distress, child behavioural problems, socio-economic disadvantage and fruit and vegetable consumption among 4-12 year-old Victorians, Australia. Health Promot Int 26, 263-275.
20. Ambrosini GL, Oddy WH, Robinson M et al. (2009) Adolescent dietary patterns are associated with lifestyle and family psycho-social factors. Public Health Nutr 12, 1807-1815.

21. Dickens E \& Ogden J (2014) The role of parental control and modelling in predicting a child's diet and relationship with food after they leave home. A prospective study. Appetite 76, 23-29.

22. Straker L, Mountain J, Jacques A et al. (2017) Cohort profile: the Western Australian Pregnancy Cohort (Raine) Study Generation 2. Int J Epidemiol 46, 1384j-1385j.

23. White SW, Eastwood PR, Straker LM et al. (2017) The Raine study had no evidence of significant perinatal selection bias after two decades of follow up: a longitudinal pregnancy cohort study. BMC Pregnancy Childbirth 17, 207.

24. Epstein NB, Baldwin LM \& Bishop DS (1983) The McMaster family assessment device. J Marital Fam Ther 9, 171-180.

25. Ambrosini GL, de Klerk NH, O'Sullivan TA et al. (2009) The reliability of a food frequency questionnaire for use among adolescents. Eur J Clin Nutr 63, 1251-1259.

26. Ambrosini GL, O'Sullivan TA, de Klerk NH et al. (2011) Relative validity of adolescent dietary patterns: a comparison of a FFQ and $3 \mathrm{~d}$ food record. Br J Nutr 105, 625-633.

27. Lassale C, Guilbert C, Keogh J et al. (2009) Estimating food intakes in Australia: validation of the Commonwealth Scientific and Industrial Research Organisation (CSIRO) food frequency questionnaire against weighed dietary intakes. J Hum Nutr Diet 22, 559-566.

28. Australian National Health and Medical Research Council \& New Zealand Ministry of Health (2006) Nutrient Reference Values for Australia and New Zealand - Dietary Fibre. Nutrient Reference Values for Australia and New Zealand. Canberra: NHMRC and MoH; available at https://www.nrv. gov.au/sites/default/files/content/n35-dietaryfibre_0.pdf (accessed March 2019).

29. R Core Team (2018) R: A Language and Environment for Statistical Computing, edition 3.5.0. Vienna: R Foundation for Statistical Computing.

30. RStudio (2018) RStudio, edition 1.1.453. Boston, MA: RStudio.

31. Olson DH (2008) Circumplex model of marital and family sytems. J Fam Ther 22, 144-167.

32. Videon TM \& Manning CK (2003) Influences on adolescent eating patterns: the importance of family meals. $J$ Adolesc Health 32, 365-373.

33. Gillman MW, Rifas-Shiman SL, Frazier AL et al. (2000) Family dinner and diet quality among older children and adolescents. Arch Fam Med 9, 235-240.

34. Sette S, Le Donne C, Piccinelli R et al. (2011) The third Italian national food consumption survey, INRAN-SCAI 2005-06 part 1: nutrient intakes in Italy. Nutr Metab Cardiovasc Dis 21, 922-932.

35. Pietinen P, Paturi M, Reinivuo H et al. (2010) FINDIET 2007 survey: energy and nutrient intakes. Public Health Nutr 13, 920-924.

36. Miller TM, Abdel-Maksoud MF, Crane LA et al. (2008) Effects of social approval bias on self-reported fruit and vegetable consumption: a randomized controlled trial. Nutr J 7, 18.

37. Prosky L, Asp NG, Furda I et al. (1985) Determination of total dietary fiber in foods and food products: collaborative study. J Assoc Off Anal Chem 68, 677-679.

38. McGill CR, Fulgoni VL \& Devareddy L (2015) Ten-year trends in fiber and whole grain intakes and food sources for the United States population: National Health and Nutrition Examination Survey 2001-2010. Nutrients 7, 1119-1130.

39. Cavadini C, Siega-Riz AM \& Popkin BM (2000) US adolescent food intake trends from 1965 to 1996. Arch Dis Child 83, $18-24$. 